NASA-TM-111354

Parametric Study of Solar Thermal Rocket Nozzle Performance

Pearson, J. B., Landrum, D. B. and Hawk, C. W., 1994

\title{
Parametric Study of Solar Thermal Rocket Nozzle Performance
}

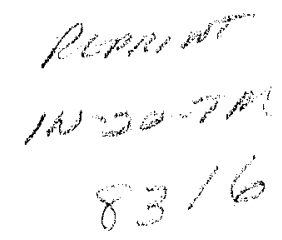

\author{
J Boise Pearson, D. Brian Landrum and Clark W. Hawk \\ Propulsion Research Center \\ The University of Alabama in Huntsville \\ Huntsville, Alabama
}

\begin{abstract}
This paper details a numerical investigation of performance losses in low-thrust solar thermal rocket nozzles. The effects of nozzle geometry on three types of losses were studied; finite rate dissociation-recombination kinetic losses, twodimensional axisymmetric divergence losses, and compressible viscous boundary layer losses. Short nozzle lengths and supersonic flow produce short residence times in the nozzle and a nearly frozen flow, resulting in large kinetic losses. Variations in geometry have a minimal effect on kinetic losses. Divergence losses are relatively small, and careful shaping of the nozzle can nearly eliminate them. The boundary layer in these small nozzles can grow to a major fraction of nozzle radius, and cause large losses. These losses are attributed to viscous drag on the nozzle walls and flow blockage by the boundary layer, especially in the throat region. Careful shaping of the nozzle can produce a significant reduction in viscous losses.
\end{abstract}

\section{INTRODUCTION}

Continued exploration and exploitation of space will require the development of more efficient and economical systems for access to and transportation through space. Due to its potential for high specific impulse $\left(\mathrm{I}_{\mathrm{sp}}\right)$, solar thermal propulsion has emerged as a candidate to meet this role. A schematic of a generic solar thermal rocket is shown in Fig. 1.

The system consists of mirrored concentrators which concentrate solar radiation on a metallic absorber. The absorber transfers the energy to a working fluid. This working fluid is heated and then thermally expanded through a contoured nozzle to produce thrust. Hydrogen, with its low molecular weight, produces high exhaust velocities and is commonly considered for the working fluid propellant. Practical system sizes and weights generaily limit solar thermal design concepts to low thrust systems.

These characteristics result in the hydrogen fueled solar thermal rocket having about twice the $I_{s p}$ of a traditional chemical system, but requiring much longer times to transfer payloads. Therefore, the primary mission envisioned for solar thermal propulsion is in an orbital transfer stage. This mission can be divided into two categories: 1.) orbital transfer between LEO and GEO; and 2.) planetary/asteroid flyby missions such as the Pluto fast flyby being developed by

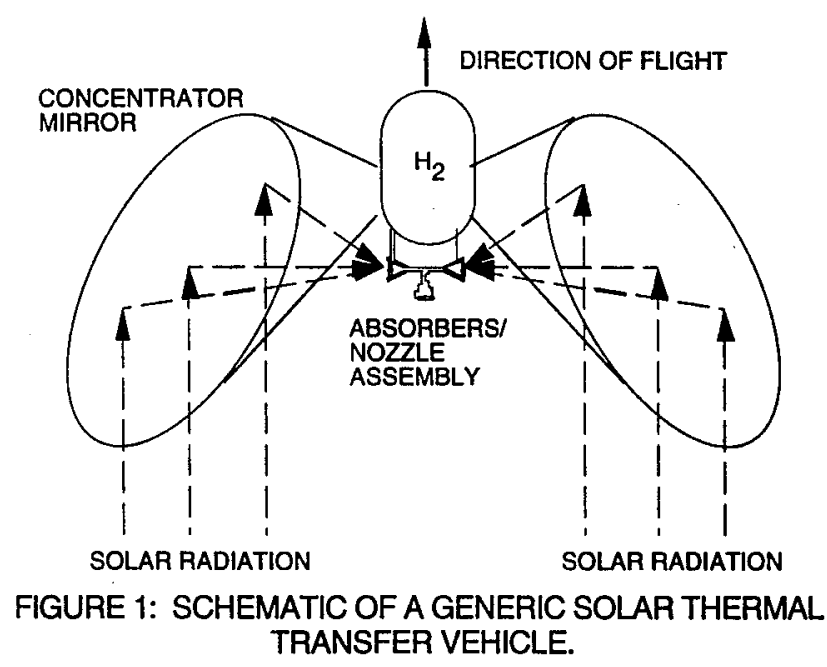

NASA. These two mission categories are differentiated by the requirements on $\Delta \mathrm{V}$, mass, and thrust of the system. The LEO/GEO transfer vehicle requires relatively low $\Delta \mathrm{V}$ 's ( $10,000 \mathrm{ft} / \mathrm{s}$, or $\sim 3,000 \mathrm{~m} / \mathrm{s})$, but high mass, and thrust on the order of $6-40 \mathrm{lbf}(25-180 \mathrm{~N})$ for Delta class vehicles, or approximately $2 \mathrm{lbf}(9 \mathrm{~N})$ for smaller vehicles such as Conestoga. The planetary flyby mission requires significantly larger $\Delta \mathrm{V}$ 's, relatively low mass, and thrust levels on the order of $1-2 \mathrm{lbf}(4-8 \mathrm{~N})$. These categories are not definitive and can significantly overlap depending on specific mission requirements.

The solar thermal system is conceptually simple, but there are significant design issues which must be addressed. These issues include the design of the concentrator, development of the absorber/heat exchanger, and the optimization of the nozzle to efficiently produce the required thrust. Research initiatives are gradually developing to examine each of these component issues. Based on preliminary system studies by the Air Force and industry, it appears that a significant drop in 
$I_{\text {sp }}(10-15 \%)$ can occur in low thrust (1-10 lbf, $\left.\sim 4-40 \mathrm{~N}\right)$ systems (Shoji, 1993). The physical causes of this performance drop must be adequately understood in order to design a practical solar thermal propulsion system.

A liquid hydrogen $\left(\mathrm{LH}_{2}\right)$ propellant can produce theoretical $I_{\text {sp }}$ values of 700-1100 seconds based on typical temperatures in the absorber/heat exchanger of $3500-6500^{\circ} \mathrm{R}(1944-3611$ $\mathrm{K})$. The temperature is primarily limited by the melt temperature of the absorber material and the cooling techniques available. At these temperatures, the liquid hydrogen is vaporized and a fraction of the gas molecules dissociate. As this hot gas thermally expands through the nozzle, it cools and recombination occurs with an associated heat release. The process of dissociation, recombination and heat release can result in a net reduction of $\mathrm{I}_{\mathrm{sp}}$ (Sutton, 1992; Kim and Stubbs, 1993). The analysis of this behavior is complicated by the fact that this may also be a thermochemically nonequilibrium process.

Other possible sources of $I_{s p}$ reduction can be attributed to viscous effects and flow divergence. The specific nozzle geometry can have a significant impact on these and the kinetic loss. Therefore, designing nozzles for optimum performance is based on careful tailoring of the geometric contours. This paper describes a fundamental study of the potential causes of $I_{s p}$ reduction in low thrust, solar thermal rocket nozzles. The main emphasis is an analytical/numerical study of how nozzle geometry impacts the viscous and thermochemical losses. The results are applicable to optimizing nozzle geometry for maximum performance.

\section{MODEL DESCRIPTION}

\subsection{Numerical Model}

The primary tool used in this investigation was the TwoDimensional Kinetics (TDK) code of Nickerson, et al. (1993). The code is a robust engineering level design and analysis tool which is used extensively in the propulsion industry. The TDK code provides various nozzle analysis options. The nozzle flow field can be solved as quasi-one-dimensional, or with an axisymmetric method of characteristics formulation. Frozen, nonequilibrium finite rate (kinetic), and equilibrium chemistry options are available. These solutions are referred to in the code and in this paper as follows: One-Dimensional Frozen (ODF), One-Dimensional Kinetic (ODK), OneDimensional Equilibrium (ODE), Two-Dimensional Frozen (TDF), Two-Dimensional Kinetic (TDK), and TwoDimensional Equilibrium (TDE).

The TDK Boundary Layer Module (BLM) uses a first-order integral method to compute nozzle performance losses due to a compressible laminar and/or turbulent boundary layer. This approach consists of using the initial method of characteristics solution to calculate the distribution of the nozzle boundary layer displacement and momentum thicknesses. The nozzle surface is then displaced by the displacement thickness to produce a new effective nozzle shape which can be re-analyzed by the method of characteristics. The BLM technique includes axisymmetric and longitudinal curvature effects. In addition, the nozzle wall can

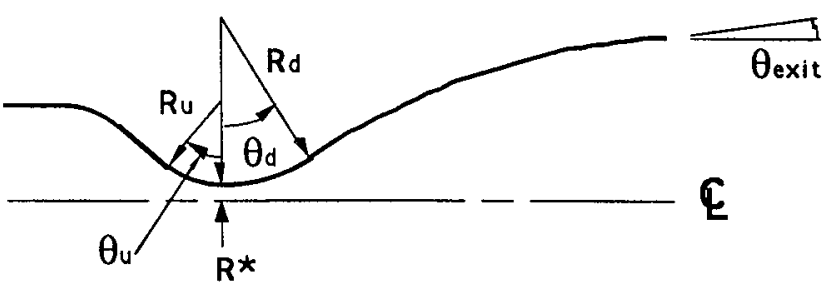

FIGURE 2: NOZZLE GEOMETRY PARAMETERS

be modeled as adiabatic or with a fixed distribution of wall temperatures or heat fluxes.

TDK provides various options for defining convergent/divergent nozzle geometries including simple conical nozzles and bell nozzles. The bell nozzle design used in this study is based on a skewed parabola as shown in Fig. 2. The nozzle transitions from a constant area combustion chamber into a conical convergent section defined by a convergence angle $\theta_{\mathrm{u}}$. The throat is defined by two circular arcs, one upstream with radius $R_{u}$, and one downstream with radius $R_{d}$. The length of the upstream arc is controlled by $R_{u}$ and the convergence angle, $\theta_{u}$. The length of the downstream arc is controlled by $R_{d}$ and the divergence angle, $\theta_{d}$. The nozzle expansion is fixed by fitting a skewed parabola to the location and slope of the downstream end of the throat and the nozzle exit. All nozzle dimensions are normalized by the throat radius, $R^{*}$.

\subsection{Nozzle Performance Analysis}

The specific impulse for a rocket is defined as

$$
I s p=\frac{F}{\dot{m}_{p} g}
$$

where $\mathbf{F}$ is the thrust, $\dot{\mathbf{m}}_{\mathbf{p}}$ is the propellant mass flow rate, and $\mathrm{g}$ is the gravitational constant. The specific impulse is therefore a measure of how efficiently a rocket produces thrust. As discussed by Sutton (1992) and O'Leary and Beck (1992), I $_{\text {sp }}$ loss mechanisms for typical bell nozzles can be divided into three primary categories: (1) chemical kinetics losses, (2) divergence losses, and (3) viscous losses. Using TDK analysis results, the effects of each of these mechanisms can be expressed as a specific impulse loss, $\Delta \mathrm{I}_{\mathrm{sp}}$.

In a solar thermal nozzle, the liquid hydrogen propellant is heated in the absorber section. This thermal energy is used to vaporize the liquid, but also produces some fraction of gas molecule dissociation. It is desirable to convert a significant fraction of this chemical energy to gas kinetic energy at the nozzle exit by recombination. The ideal case is for the gas to attain chemical equilibrium at every point in the nozzle flow field. However, the expanding flow can accelerate gas molecules so rapidly that their residence time in the nozzle does not permit full equilibrium to be reached. This state of thermochemical nonequilibrium results in a loss in efficiency. 
A measure of this effect can be calculated as the difference in the TDK one-dimensional equilibrium and one-dimensional kinetics (nonequilibrium) solutions.

$$
\Delta I_{\mathrm{sp}(\mathrm{KIN})}=\mathrm{I}_{\mathrm{sp}(\mathrm{ODE})}-\mathbf{I}_{\mathrm{sp}(\mathrm{ODK})}
$$

Theoretically, the kinetics loss can be minimized by increasing the residence time of the fluid in regions where the temperature is decreasing and the pressure is still high enough to promote recombination.

Divergence losses occur when portions of the exit flow are not parallel to the nozzle axis. This results in a radial component of momentum which reduces the net axial thrust. A measure of this effect can be obtained by comparing the TDK one-dimensional kinetics solution to the two-dimensional kinetics solution.

$$
\Delta \mathbf{I}_{\mathrm{sp}(\mathrm{DIV})}=\mathbf{I}_{\mathrm{sp}(\mathrm{ODK})}-\mathbf{I}_{\mathrm{sp}(\mathrm{TDK})}
$$

The divergence loss can generally be minimized by carefully shaping the nozzle as discussed, for example, by Rao (1960) Lengthening the nozzle is also an alternative, though this may increase the nozzle weight to a point that it is not practical from mission considerations.

The last loss mechanism is due to two types of viscous effects. The first is due to viscous drag on the nozzle walls. The second comes from flow blockage due to boundary layer growth, especially in the throat region. A measure of these combined effects can be defined by the difference in the TDK inviscid two-dimensional kinetics and boundary layer solutions

$$
\Delta I_{\mathrm{sp}(\mathrm{VIS})}=\mathbf{I}_{\mathrm{sp}(\mathrm{TDK})}-\mathbf{I}_{\mathrm{sp}(\mathrm{BLM})}
$$

The shorter the nozzle, the less surface area for viscous drag and heat transfer to act on. Therefore, the viscous loss is minimized by reducing the nozzle's wetted surface. The blockage effect can be significant for small throat solar thermal nozzles. Its impact can be reduced by increasing the throat diameter. A more detailed study of this blockage effect will be the subject of future work.

The overall nozzle performance loss is the sum of these individual losses. But, minimizing one of the individual losses may produce a corresponding increase in the loss from another mechanism. Therefore, maximizing overall nozzle efficiency is a process of compromise between each of the loss mechanisms. This paper describes the impact of geometric parameters on these loss mechanisms with the ultimate goal of developing guidelines for optimizing the total nozzle efficiency.

\subsection{Nozzle Design Constraints}

A baseline nozzle configuration and operating conditions were provided by Rocketdyne for this study. A sketch of the baseline nozzle with the geometric parameters for the TDK model of the baseline are shown in Fig. 3. Plenum chamber conditions were $50 \mathrm{psia}$ and $6100^{\circ} \mathrm{R}(0.345 \mathrm{MPa}$ and $3611 \mathrm{~K})$. This temperature is the upper limit for a hafnium-carbide

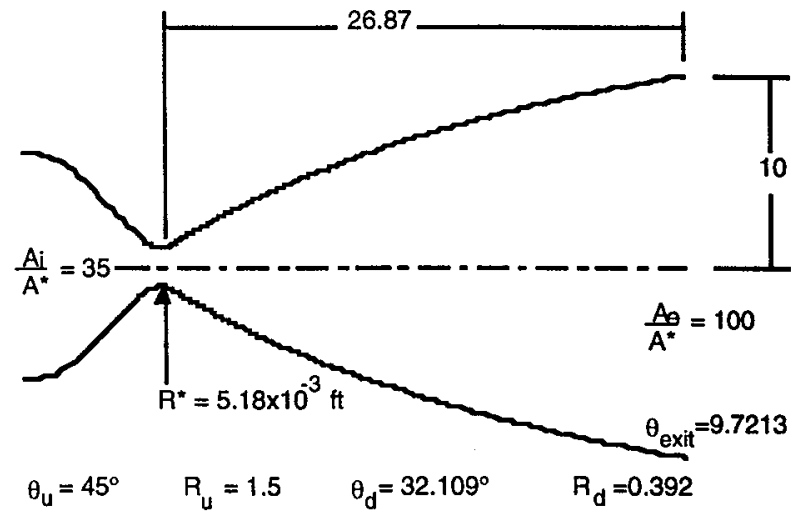

\section{FIGURE 3: BASELINE NOZZLE. ALL DIMENSIONS NORMALIZED BY THROAT RADIUS, $R^{\star}$.}

absorber. The chamber pressure is limited by the yield stress of this material at the elevated temperature (Shoji, 1993, McClanahan, et al. 1994). These conditions produce an equilibrium molecular hydrogen mass fraction of 0.878 . The nozzle throat area is $8.43 \times 10^{-5} \mathrm{ft}^{2}\left(7.83 \times 10^{-6} \mathrm{~m}^{2}\right)$. The baseline area ratio is 100 . This area ratio and chamber conditions produce approximately $1.0 \mathrm{lbf}$ nominal thrust. In this study, the nozzle throat diameter, contraction area ratio, expansion area ratio and chamber conditions were all held constant. The four parameters that describe the throat contour, $\mathrm{R}_{\mathrm{u}}, \mathrm{R}_{\mathrm{d}}, \theta_{\mathrm{u}}$, and $\theta_{\mathrm{d}}$. were varied independently. This combination of fixed and variable parameters allowed the convergent region cone angle and length to vary (with $\theta_{u}$ ), and allowed the divergent section contour (skewed parabola) to vary as well. The divergent section length was maintained constant at $0.1392 \mathrm{ft}(0.0424 \mathrm{~m})$. The boundary layer was allowed to transition from laminar to turbulent, with a criteria for transition to turbulence of $\operatorname{Re} \geq 400$, with Reynold's Number based on momentum thickness. For all cases run in this study the boundary layer remained laminar.

\section{HYDROGEN DISSOCIATION RATE STUDY}

In a solar thermal rocket, liquid hydrogen is vaporized and partially dissociated. The recovery of kinetic energy through the exothermic recombination of hydrogen atoms increases the $I_{s p}$ of the nozzle. As discussed, nonequilibrium (finite rate) hydrogen kinetics can significantly affect this process. Assuming a pure hydrogen propellant and neglecting radiation effects, the exothermic recombination of hydrogen is governed by two reactions

$$
\mathbf{H}+\mathbf{H}+\mathbf{M}_{\mathbf{i}} \Leftrightarrow \mathbf{H}_{2}+\mathbf{M}_{\mathbf{i}}
$$

where $\mathbf{M}_{\mathbf{i}}$ is either an atomic $(\mathbf{H})$ or molecular $\left(\mathbf{H}_{2}\right)$ third- 
TABLE 1: HYDROGEN KINETIC REACTHN RATE COEFFICIENTS (EQ. 6)

\begin{tabular}{|l|c|c|c|c|}
\hline Reference & $\mathbf{M}_{\mathbf{i}}$ & $\mathbf{A}_{\mathbf{i}}$ & $\mathbf{N}_{\mathbf{i}}$ & $\mathbf{B}_{\mathbf{i}}$ \\
\hline \hline $\begin{array}{l}\text { Cohen \& } \\
\text { Westberg (1983) }\end{array}$ & & & & \\
\hline Low & $\mathrm{H}_{2}$ & $0.5 \times 10^{17}$ & -0.6 & 0 \\
\hline Nominal & & $1.02 \times 10^{17}$ & -0.6 & 0 \\
\hline High & & $2.0 \times 10^{17}$ & -0.6 & 0 \\
\hline Low & $\mathrm{H}$ & $1.0 \times 10^{15}$ & 0 & 0 \\
\hline Nominal & & $3.19 \times 10^{15}$ & 0 & 0 \\
\hline High & & $1.0 \times 10^{16}$ & 0 & 0 \\
\hline \hline NASP (1990) & $\mathrm{H}_{2}$ & $1.83 \times 10^{18}$ & -1.0 & 0 \\
\hline & $\mathrm{H}$ & $7.3 \times 10^{17}$ & -1.0 & 0 \\
\hline \hline TDK Standard & $\mathrm{H}_{2}$ & $2.56 \times 10^{18}$ & -1.0 & 0 \\
\hline & $\mathrm{H}$ & $1.6 \times 10^{19}$ & -1.0 & 0 \\
\hline \hline Baulch & $\mathrm{H}_{2}$ & $2.98 \times 10^{16}$ & -0.45 & -1.5 \\
\hline & $\mathrm{H}^{17}$ & $9.43 \times 10^{16}$ & -0.34 & 1.2 \\
\hline \hline Wetzel \& \\
Solomon (1993) & & & & \\
\hline Low & $\mathrm{H}_{2}$ & $2.7 \times 10^{16}$ & -0.58 & 0 \\
\hline Nominal & & $9.7 \times 10^{16}$ & -0.6 & 0 \\
\hline High & & $2.0 \times 10^{16}$ & 0 & 0 \\
\hline Low & $\mathrm{H}$ & $7.4 \times 10^{16}$ & -0.63 & 0 \\
\hline Nominal & & $9.1 \times 10^{16}$ & -0.37 & 0 \\
\hline High & & $6.0 \times 10^{16}$ & 0 & 0 \\
\hline \hline FDNS & $\mathrm{H}_{2}$ & $5.0 \times 10^{15}$ & 0 & 0 \\
\hline & $\mathrm{H}$ & $5.0 \times 10^{15}$ & 0 & 0 \\
\hline
\end{tabular}

Rate $\left(k_{i}\right)$ Units $=\mathrm{cm}^{6} / \mathrm{Mole}^{2}$-sec

body. The rate of each reaction can be written in the Arrhenius form

$$
\mathbf{k}_{\mathbf{i}}=\mathbf{A}_{\mathbf{i}} \mathbf{T}^{N_{i}} \mathbf{e}^{\left(-\frac{1000 B_{i}}{R T}\right),}
$$

where $\mathbf{T}$ is temperature and $\mathbf{R}$ is the universal gas constant. Variation in experimental results have produced uncertainties in these reaction rates which are as much as two orders of magnitude (Wetzel and Solomon, 1993; Gerrish and Doughty, 1993). This uncertainty may have a significant impact on the prediction of solar thermal rocket performance. The research literature offers a range of hydrogen third-body recombination rates in the temperature and pressure range of this study. These rates are summarized in Table 1 in the form of the constants for the Arrhenius rate of Eq. (6).

Cohen and Westberg (1983) surveyed the literature and defined representative low, nominal and high rates. Research into NASP propulsion technologies resulted in a survey of

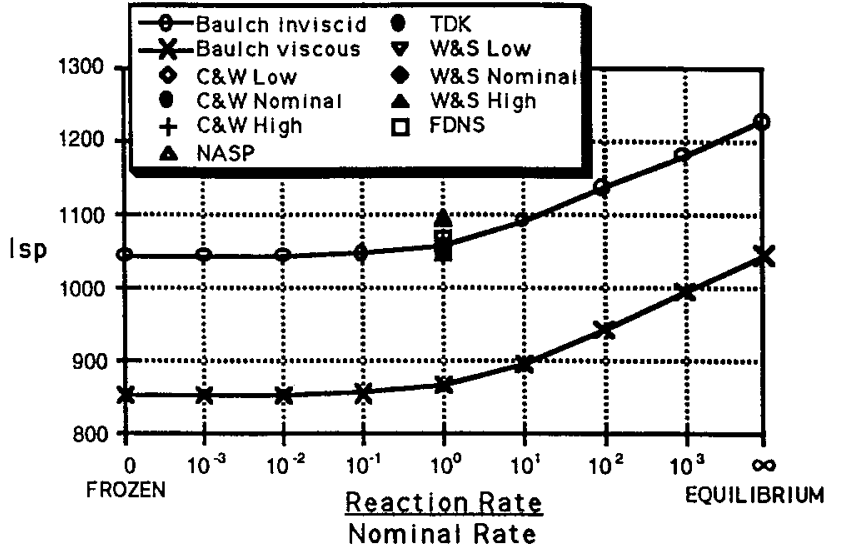

FIGURE 4: SENSITIVITY OF SPECIFIC IMPULSE TO REACTION RATE.

hydrogen reaction rates and establishment of a standard set (Oldenborg, 1990). The TDK code (Nickerson, et al. 1993) and the NASA developed Finite Difference Navier-Stokes (FDNS) code (Chen, 1993) provide internal default rates. Rocketdyne provided baseline rates based on the work of Baulch, et al. (1972). Finally, Wetzel and Solomon (1993) studied the impact of rate uncertainty on the performance of high thrust $(\sim 10,000 \mathrm{lbf}$ or $-45,000 \mathrm{~N})$ nuclear thermal rockets. They also defined a set of high, nominal and low rates. The TDK code was run with each of these rates and the baseline nozzle under inviscid conditions. The resultant $\mathbf{I}_{\mathbf{s p}}$ values were calculated and are plotted in Fig. 4 as nominal rates $\left(\mathrm{k} \times 10^{0}\right)$. The rate uncertainty produces an uncertainty in $\mathrm{I}_{\mathrm{sp}}$ of approximately 50 seconds. Neglecting the Wetzel and Solomon high rates, the Baulch et al. (1972) rates produce an $I_{s p}$ which is approximately the average of the values calculated using the other rates. Therefore, these rates were chosen as the nominal rates for the rest of this investigation.

The TDK code was next used to calculate the impact on the baseline nozzle $I_{\mathrm{sp}}$ of increasing and decreasing the nominal rates by successive factors of 10 . The baseline nozzle was also run with frozen and equilibrium chemistry specified. The analysis was made for both inviscid and viscous cases. The resultant curves are also shown in Fig. 4 . These results illustrate that the nominal rates produce near frozen chemistry in the flow, indicating that most of the thermal energy used to dissociate the $\mathrm{H}_{2}$ is not recovered in the nozzle, thereby reducing performance. Finite rate kinetics cause an $\mathbf{I}_{\mathbf{s p}}$ loss of approximately $170 \mathrm{sec}(\sim 13.8 \%)$ from the ideal equilibrium chemistry value. It should be noted that the magnitude of the kinetic losses is strongly dependent on the amount of dissociation in the chamber, and is therefore strongly dependent on chamber temperature. Lower chamber temperatures reduce kinetic effects significantly. The magnitude of performance lost due to viscous effects $(210 \mathrm{sec}$, $\sim 18 \%$ ) is comparable to the kinetics loss. 


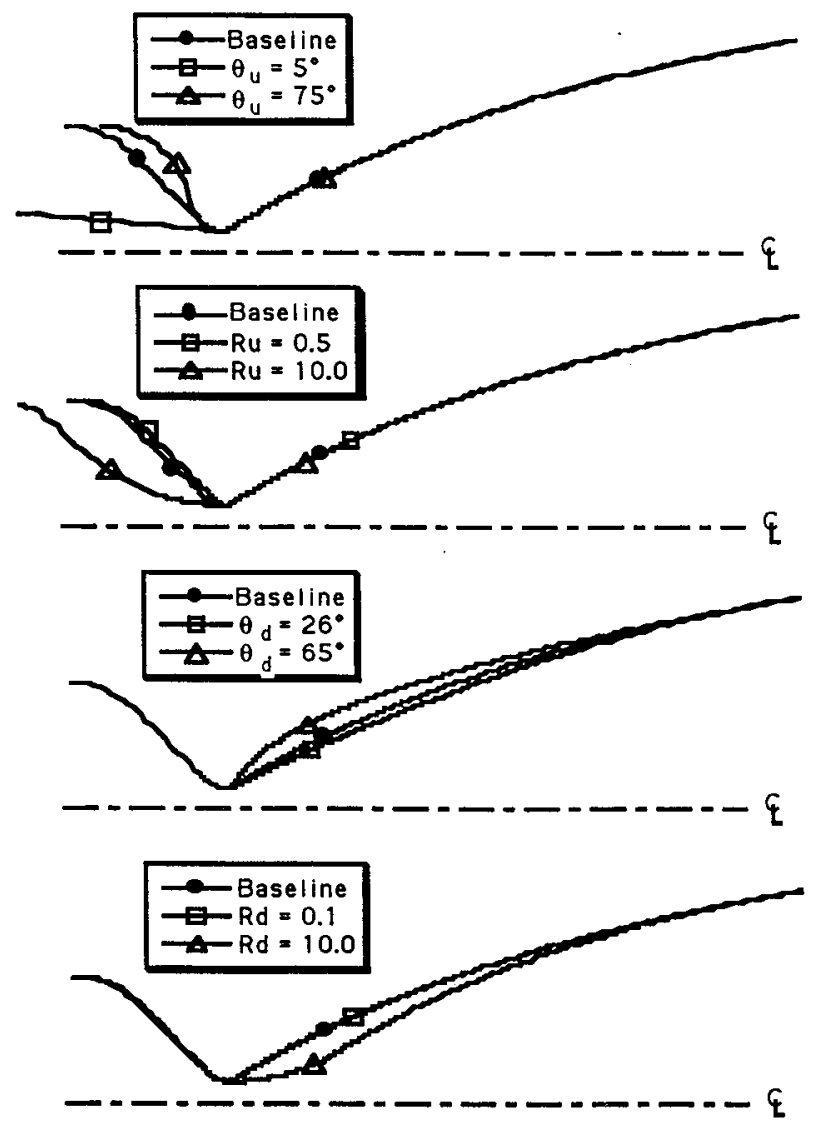

FIGURE 5: REPRESENTATIVE NOZZLE CONTOURS.

\section{EFFECTS OF GEOMETRIC PARAMETERS ON PERFORMANCE}

Parametric variation of the nozzle geometry was done with three goals in mind. First, the primary geometric parameters (i.e. length, throat diameter, area ratio) and inlet (plenum) conditions were held constant so that thrust levels for the various configurations were comparable. Second, recombination was to be maximized to increase $I_{\mathrm{sp}}$. Third, viscous effects should be minimized to increase Isp.

Results obtained from studies of nuclear thermal rockets (Wetzel and Solomon, 1993; Gerrish and Doughty 1993) indicate that lengthening of the throat region encourages recombination. Therefore, the four parameters that define the throat contour, $R_{u}, R_{d}, \theta_{u}$, and $\theta_{d}$, were varied independently. Figure 5 shows representative nozzle contours produced by the variation of these four throat parameters. The nozzle length was essentially maintained constant, except for cases of small $\theta_{u}$ and large $R_{u}$ where the convergent section length is significantly longer than for the other nozzles. These longer nozzles do not maintain the original geometry, but are presented for completeness.

A matrix of nozzle configurations were analyzed by the TDK code to determine the impact of geometry on $\mathrm{I}_{\mathrm{sp}}$. The results

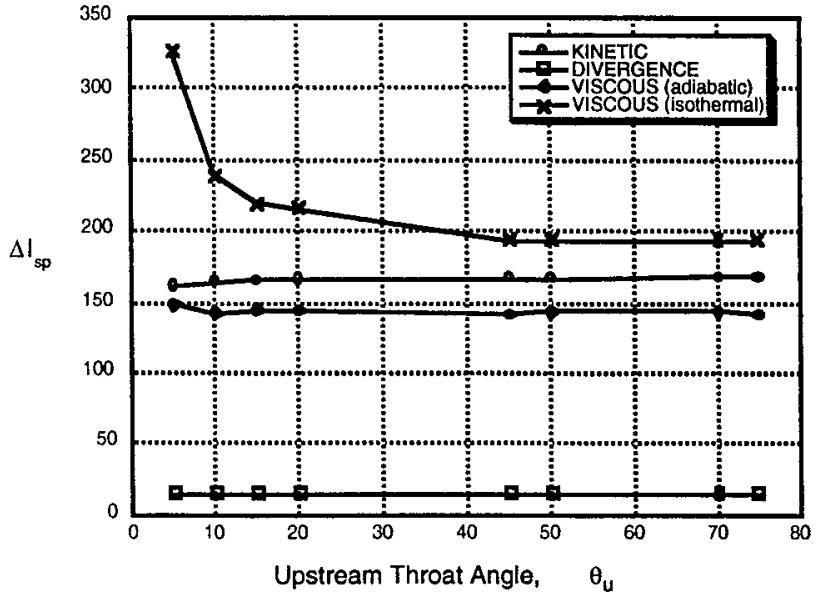

FIGURE 6a: PERFORMANCE TRENDS FOR VARIATION IN $\theta_{\mathrm{u}}$.

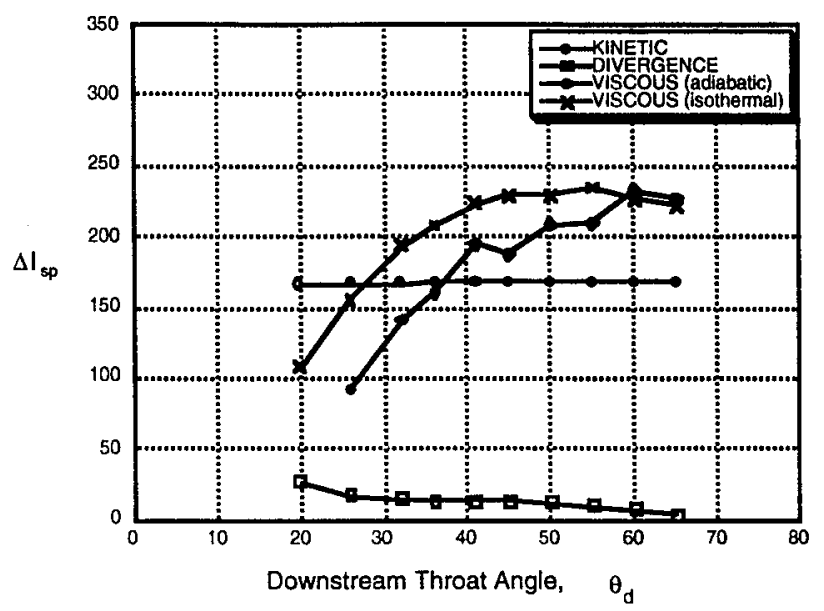

FIGURE 6b: PERFORMANCE TRENDS FOR VARIATION IN $\theta_{d}$.

are shown in Figs. 6a - 6d. The ideal $\mathrm{I}_{\mathrm{sp}}$ of 1241 seconds was determined by a one-dimensional equilibrium (ODE) TDK analysis. The effect of each geometric parameter on the kinetic, divergence and viscous losses is expressed in terms of the $\Delta \mathrm{I}_{\mathrm{sp}}$ definitions described in section 2.2. Improved performance is indicated by smaller values of $\Delta \mathrm{I}_{\mathrm{sp}}$. For the viscous calculations, both an adiabatic and constant temperature wall were analyzed. The constant temperature wall condition was set to $2460^{\circ} \mathrm{R}$. This value was determined based on inclusion of radiation cooling through the exit plane and no liquid wall cooling.

The $\mathrm{I}_{\mathrm{Sp}}$ loss due to finite rate kinetics (ODE-ODK) is between 160 and 170 seconds. In these small nozzles this is primarily due to the relatively short stay times in flow regions 


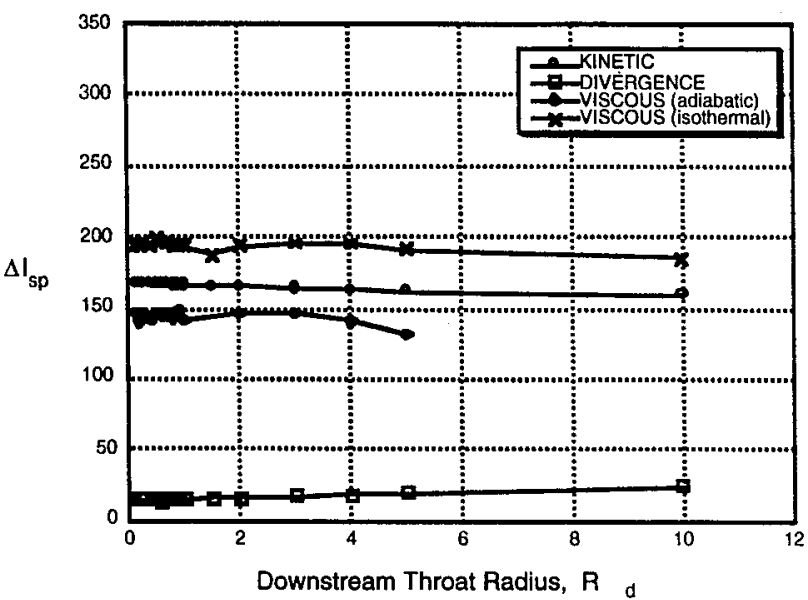

FIGURE 6c: PERFORMANCE TRENDS FOR VARIATION IN Rd.

where the temperature is decreasing and the pressure is still high enough to promote recombination. The loss is therefore minimized by increasing the throat length through decreasing the converging and diverging angles or by increasing the upstream and downstream radii. But, as shown in Figures 6a$6 \mathrm{~d}$, the kinetic loss is nearly insensitive to throat geometry. This result is not surprising since the reaction rates produce nearly frozen chemistry as shown in Fig. 4.

This point is further illustrated in Fig. 7 where plots of atomic hydrogen mass fraction versus axial area ratio variation are shown for several representative geometries. These geometries were chosen to show the entire range of mass fraction variation found in the study. This figure indicates that the flow chemistry effectively freezes at approximately the same area ratio of 3:1 (and consequently close to the same pressure) for all nozzle geometries in this study. For the best case $\left(R_{\mathbf{u}}=10\right)$ only $1 \%$ of the mass is converted from atomic to molecular hydrogen.

Divergence losses due to two-dimensional flow (ODK-TDK) vary between 3 and 35 seconds. Both upstream geometry parameters have no impact on this loss, as expected. Increasing the downstream diverging angle, $\theta_{\mathrm{d}}$, to $65^{\circ}$ (see Fig. 6b) almost eliminates the divergence loss. Higher values of $\theta_{\mathrm{d}}$ expand the flow quickly and allow more time for axial turning of the flow, while the lowest values of $\theta_{d}$ produce an almost conical nozzle, which is dominated by divergence losses. A lesser reduction in divergence loss is obtained by decreasing the downstream radius of curvature (see Fig. 6c). These effects somewhat compensate for the kinetic losses.

Although modifications of the four geometric throat parameters have a relatively modest impact on the kinetic and divergence losses, the viscous losses can be significantly affected. In general, geometric changes which increase the wetted nozzle surface area increase the viscous losses. Viscous losses for the isothermal wall are typically larger than for the adiabatic wall. As shown in Fig. 6a, decreasing the upstream convergence angle produces a very long convergent section. This allows greater boundary layer growth and provides more

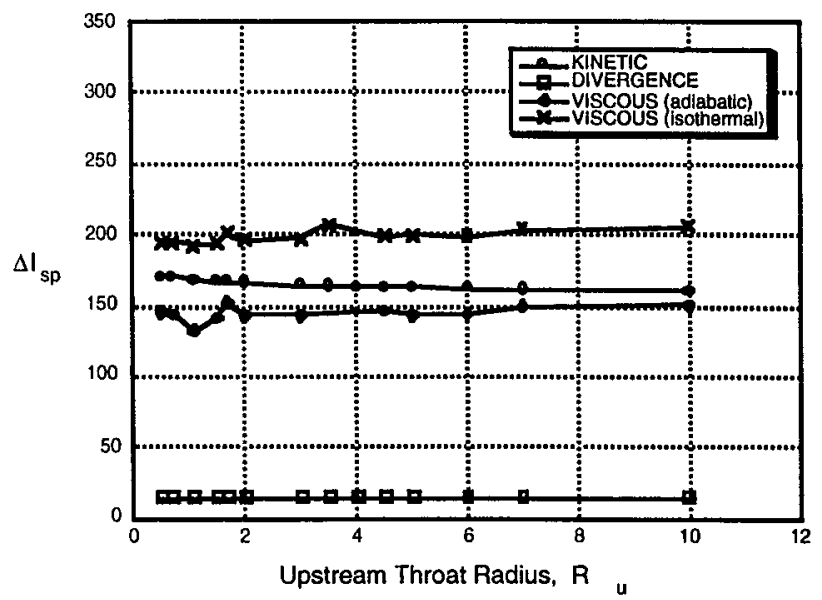

FIGURE 6d: PERFORMANCE TRENDS FOR VARIATION IN Ru.

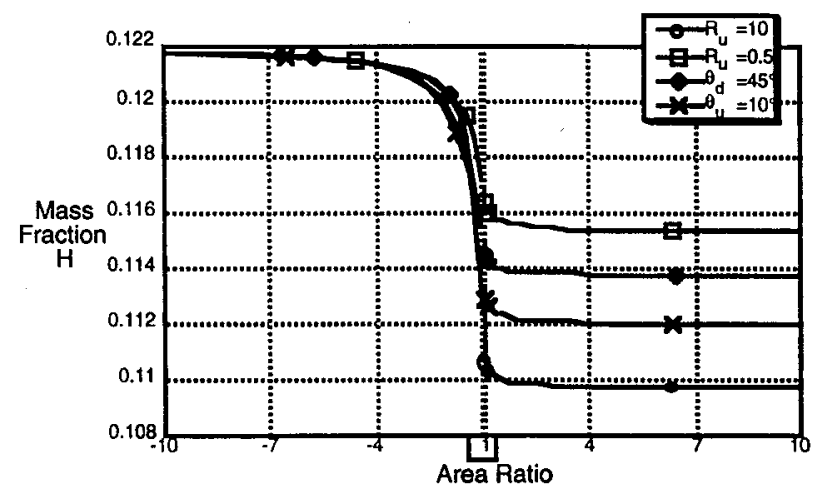

FIGURE 7: REPRESENTATIVE AXIAL VARIATIONS IN MASS FRACTION OF $\mathrm{H}$.

surface area for heat transfer losses. For $\theta_{\mathrm{u}} \geq 45^{\circ}$, the convergence angle does not significantly affect the isothermal wall viscous loss. The adiabatic wall viscous loss decreases slightly with decreasing $\theta_{\mathrm{u}}$. Decreasing the downstream divergence angle, $\theta_{\mathrm{d}}$, from $65^{\circ}$ to $20^{\circ}$ decreases the viscous losses by as much as 130 seconds of a total of 230 seconds (see Fig. 6b). This performance gain is offset slightly by the increase in divergence losses with decreasing $\theta_{\mathrm{d}}$.

Increasing the downstream throat radius of curvature, $R_{d}$, decreases the viscous $I_{s p}$ loss by approximately 10 seconds. This gain is offset by the opposite trend in divergence loss. Increasing the upstream throat radius of curvature, $R_{u}$, increases the viscous loss by approximately 10 seconds. But, this decrease in performance is offset by a comparable reduction in kinetic losses with increasing $R_{u}$. 


\section{CONCLUSIONS}

Careful shaping of a nozzle within fixed constraints can potentially result in large gains in performance. This paper has dealt with the effect of nozzle geometry on three types of performance losses; finite rate dissociation-recombination kinetic losses, two-dimensional axisymmetric divergence losses, and compressible viscous boundary layer losses.

The dissociation/recombination chemistry in these small nozzles was found to be nearly frozen. The lack of recombination caused an $I_{\mathbf{s p}}$ loss of $\sim 150$ seconds when compared to equilibrium chemistry. Geometry variations had little effect on the chemistry. The effect may be greater and is worth investigating for larger nozzles.

Divergence losses are well understood and documented in the literature. These losses accounted for no more than 30 seconds of $I_{s p}$, and can be nearly eliminated through careful nozzle shaping.

The boundary layer was the largest contributor to performance loss $\left(\Delta \mathrm{I}_{\mathrm{sp}} \sim 200-300\right.$ seconds $)$, but also showed the only significant reduction of losses with geometry variations. These losses were largely insensitive to upstream throat variations and downstream throat radius. The major gains were seen with variations in downstream throat angle, which largely determines the rate of expansion of the flow. The effect of the boundary layer was reduced when the downstream throat angle was low and the flow was allowed to expand more gradually. An $I_{S p}$ gain of $\sim 130$ seconds was predicted by TDK when $\theta_{\mathrm{d}}$ was reduced from the baseline value of $45^{\circ}$ to $20^{\circ}$.

There is some concern with the uncoupled boundary layer solver and its applicability to this problem. The assumption of an uncoupled inviscid core and viscous boundary layer solution is approximate, and generally deemed appropriate for 'thin' boundary layers. The boundary layer in the small solar thermal nozzle occupies a large portion of the flow path. Further study needs to be done using more accurate methods or fully coupled solutions (i.e. CFD) to determine the magnitude of these trends.

The numerical results of this study provide useful guidelines for the optimal design of low thrust solar thermal nozzles. All of these analyses should be verified with experimental data. A priority of future work should be placed on the design of appropriate experiments to validate these results.

\section{ACKNOWLEDGMENTS}

The authors would like to acknowledge the sponsorship of this work under contract \#R34KEZ93033655Z from Rockwell International - Rocketdyne Division. Mr. Jim Shoji served as technical monitor. The authors would also like to thank Jim McClanahan and Anthony Tu, also from Rocketdyne for their helpful comments.

\section{REFERENCES}

Baulch, D. L., Drysdale, D. D., Horne, D. G., and Lloyd, A. C., 1972, Evaluated Kinetic Data for High Temperature Reactions, Vol. 1, pp. 261-326, Butterworths, London.
Chen, Y.S., 1993, "FDNS, A General Purpose CFD Code, User's Guide, Version 3," Engineering Sciences, Inc., TR-9301, May 1, 1993.

Cohen, N., and Westberg, K. R., 1983, "Chemical Kinetic Data Sheets for High-Temperature Chemical Reactions," Journal of Physical Chemistry Reference Data, Vol. 12, No. 3, pp. 531-590.

Gerrish, Jr, H. P., and Doughty, G. E., 1993, "Performance Assessment of Low Pressure Nuclear Thermal Propulsion," NASA TM-108433, George C. Marshall Space Flight Center.

Kim, S., and Stubbs, R., 1992, "Numerical Study of Low Pressure Nuclear Thermal Rockets," AIAA Paper No. 92-3815, AIAA/SAE/ASME/ASEE 28th Joint Propulsion Conference and Exhibit, July 6-8, Nashville, TN.

McClanahan, J., Tu, A., and Shoji, J., 1994 Personal Communications Concerning Solar Thermal Propulsion Systems, Jan.-Oct.

Nickerson, G. R., Berker, D. R., Coats, D. E., and Dunn, S. S., 1993, "Two-Dimensional Kinetics (TDK) Nozzle Performance Computer Program, Vol. I - Engineering Methods," NASA8-39048.

Oldenborg, R., 1990, "Hypersonic Combustion KineticsStatus Report of the Rate Constant Committee, NASP HighSpeed Propulsion Technology Team," NASP Technical Memorandum 1107.

O'Leary, R. A., and Beck, J. E., 1992, "Nozzle Design," Threshold, Spring, pp. 34-43.

Rao, G. V. R., 1960, "Approximation of Optimum Thrust Nozzle Contour," ARS Journal, Vol. 30, no. 6, June 1960, p. 561 .

Shoji, J., 1993, "Solar Thermal Propulsion Transfer Stage (STPTS)," Rockwell International - Rocketdyne Division Briefing Presentation.

Wetzel, K., and Solomon, W., 1993, "Effect of the Uncertainty in Hydrogen Recombination Kinetics on NTR Performance Prediction" AIAA Paper No. 93-2499, 29th AIAA/ASME/SAE/ASEE Joint Propulsion Conference, June 28-30, Monterey, CA. 